\title{
Prediction and follow-up of risk factors for severe SARS-CoV-2 pneumonia and application of CT visual scoring
}

\author{
Yingjian Ye ${ }^{\mathrm{a}, 1}$, Xiaxia Wu ${ }^{\mathrm{b}, 1}$, Xiumei $\mathrm{Li}^{\mathrm{a}}$, Chunmei $\mathrm{Xu}^{\mathrm{a}}$, Qingpeng Wang ${ }^{\mathrm{c}}$, Wenhuan Yuan ${ }^{\mathrm{d}}$, \\ Li Zhang ${ }^{\mathrm{f}}$, Huan $\mathrm{Li}^{\mathrm{e}}$, Lishi Zheng ${ }^{\mathrm{e}}$, Qiongxia $\mathrm{Li}^{\mathrm{a}}$, Zhibing He ${ }^{\mathrm{b}, *}$ and Bo Liu ${ }^{\mathrm{a}, *}$ \\ ${ }^{a}$ Department of Gastroenterology and endoscopy, Xiangyang No. 1 People's Hospital Affiliated to Hubei \\ University of Medicine, Xiangyang, Hubei 441000, China \\ ${ }^{\mathrm{b}}$ Department of Radiology, Xiangyang No. 1 People's Hospital Affiliated to Hubei University of \\ Medicine, Xiangyang, Hubei 441000, China \\ ${ }^{\mathrm{c}}$ Department of Cardiac Surgery, Union Hospital, Tongji Medical College, Huazhong University of \\ Science and Technology, Wuhan, Hubei 430022, China \\ ${ }^{\mathrm{d}}$ Department of Radiology, Baotou First Affiliated Hospital, Baotou Medical College of Inner Mongolia \\ University of Science and Technology, Baotou, Inner Mongolia Autonomous Region 0472, China \\ ${ }^{\mathrm{e}}$ Center for Internal Medicine and Medical Statistics, Xiangyang No. 1 People's Hospital Affiliated to \\ Hubei University of Medicine, Xiangyang, Hubei 441000, China \\ ${ }^{\mathrm{f}}$ Department of Nuclear Medicine, Union Hospital, Tongji Medical College, Huazhong University of \\ Science and Technology, Wuhan, Hubei 430022, China
}

\begin{abstract}
.
BACKGROUND: The SARS-CoV-2 pneumonia infection is associated with high rates of hospitalization and mortality and this has placed healthcare systems under strain. Our study provides a novel method for the progress prediction, clinical treatment and prognosis of NCP, and has important clinical value for timely treatment of severe NCP patients.

OBJECTIVE: To summarize the clinical features and severe illness risk factors of the patients with novel coronavirus pneumonia (NCP), in order to provide support for the progression prediction, clinical treatment and prognosis of NCP patients.

MATERIALS AND METHODS: A total of 196 NCP patients treated in our hospital from January 25, 2020 to June 21, 2020 were divided into the severe group and the mild group. The clinical features of the two groups were analyzed and compared. The risk factors were explored by using multivariate logistic regression, and the receiver operating characteristic (ROC) curve was obtained. The correlations of the risk factors with the prognosis of NCP were investigated combined with the lung function test. RESULTS: The primary clinical symptoms of 196 cases of NCP included fever in 167 cases $(85.2 \%)$ and cough in 121 cases $(61.73 \%)$. The chest computed tomography (CT) scans of the 178 cases $(90.81 \%)$ showed a typical ground-glass opacification. In 149 cases, the lymphocyte count was decreased, while the levels of creatine kinase (CK), lactate dehydrogenase (LDH), c-reactive protein (CRP), erythrocyte sedimentation rate (ESR) and D-dimer (D-D) increased. 44 cases (22.45\%) were found to be severely ill. The multivariate logistic regression analysis demonstrated that age, underlying disease, length of hospital stay, body mass index (BMI), LDH, chest CT visual score, absolute lymphocyte count (ALC) and CRP were risk factors for severe
\end{abstract}

\footnotetext{
${ }^{1}$ Yingjian Ye and Xiaxia Wu contributed equally to this study.

${ }^{*}$ Corresponding authors: Zhibing He, Department of Radiology, Xiangyang No. 1 People's Hospital Affiliated to Hubei University of Medicine, Xiangyang, Hubei 441000, China. Tel.: +86 7103420272; E-mail: 6692409528@qq.com. Bo Liu, Department of Gastroenterology and Endoscopy, Xiangyang No. 1 People's Hospital Affiliated to Hubei University of Medicine, Xiangyang, Hubei 441000, China. Tel.: +86 7103420030; E-mail: liuxyyy2015@163.com.
} 
illness. The most valuable indicator for the prediction of severe NCP was the chest CT visual score. With a CT visual score of $<$ 8.97 as the cut-off, its prediction of severe illness gave a sensitivity of $93.22 \%$ and a specificity of $71.53 \%$, and it was negatively correlated with the prognostic diffusing capacity of the lungs for carbon monoxide (DLCO\%), with a coefficient of 0.702 .

CONCLUSIONS: The primary symptoms of NCP were fever and dry cough, followed by a rapid disease progression. The chest CT visual score was found to exhibit a key role in the prediction of severe illness and prognosis, and served as an early warning indicator of severe NCP. The CT visual score opens up a new window for pre-critical research of NCP.

Keywords: Novel coronavirus, COVID-19, pneumonia, CT visual scoring, risk factors, prognosis

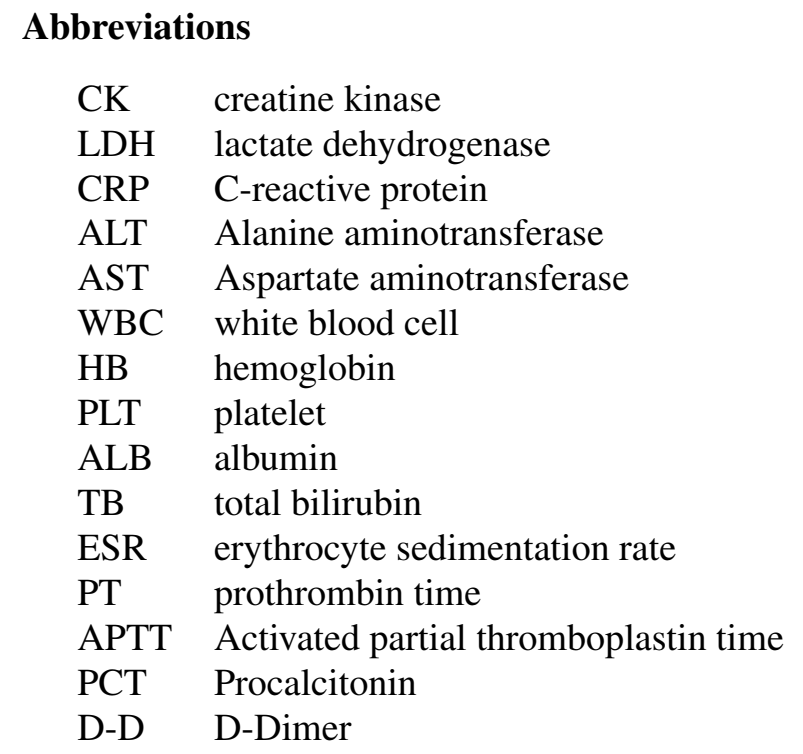

\section{Introduction}

Since a large-scale pneumonia outbreak of unknown etiology in Wuhan, China in December 2019, the Wuhan Institute of Virology, Chinese Academy of Sciences successfully isolated a new type of coronavirus from the culture of secretions of novel coronavirus pneumonia (NCP) patients and identified its genome sequence on January 7, 2020. The sequence of this virus is homologous to the severe acute respiratory syndrome (SARS) and Middle East respiratory syndrome (MERS). The virus has a round or elliptic envelope, often pleomorphic form, with a diameter of 60-140 nm [1-3]. On February 11, 2020 the World Health Organization (WHO) named it SARS-CoV-2. The virus caused multiple system infections in various animal species, and mainly respiratory infections in humans. The NCP caused by SARS-CoV-2 is highly contagious, and it has spread rapidly to 212 countries including the United States, Brazil, and Italy. Currently, the cumulative number of infections runs above 13.5 million, and the death toll is over 600,000 . The SARS-CoV-2 quickly triggered the global public health emergencies and has caused a heavy health and economic burden on the world [4,5].

There have been more than 300 reports reporting the imaging features, clinical features and treatment options of NCP on PubMed. The literature mostly summarized the imaging diagnosis, disease development and clinical outcomes of NCP, and rarely analyzed its risk factors, thus providing limited guidance to clinical treatment [6,7]. In this pursuit, the present study explored the imaging and clinical data of 196 cases of NCP, and added the CT visual score to multivariate logistic regression for the first time and 
obtained encouraging research results. We successfully predicted the risk factors for severe NCP, including the length of hospital stay, body mass index (BMI), and chest CT visual score. Finally, in combination with the receiver operating characteristic (ROC) curve, we obtained the cut-off value for the CT visual score, and confirmed that the CT score was negatively correlated with the lung function indicators like forced vital capacity (FVC)\%, total lung capacity (TLC)\%, and diffusing capacity of lung for carbon monoxide (DLCO)\%. Our study provides a novel method for the progress prediction, clinical treatment and prognosis of NCP, and has important clinical value for timely treatment of severe NCP patients.

\section{Materials and methods}

\subsection{General information}

The clinical, serum biochemical tests and imaging data of 196 subjects of laboratory-confirmed NCP with positive SARS-CoV-2 nucleic acid tested by RT-PCR were collected in our hospital from January 25, 2020 to June 21,2020 . There were 137 cases $(69.89 \%$ ) with positive nucleic acid results for the first round of screening, and 59 cases $(30.11 \%)$ with positive results for the second or further round. The age range of patients was $19 \sim 83$, with an average of $(51.7 \pm 12.98)$ years old, including 107 males and 89 females (1.2:1). 117 patients (59.69\%) exhibited a contact history with confirmed NPC patients, and 79 cases had no clear epidemiologic links. The incubation period was $1 \sim 20$ days, with an average of $(8.09 \pm 4.09)$ days. 80 cases $(40.82 \%)$ had underlying diseases, such as chronic bronchitis, hypertension, coronary heart disease, diabetes, chronic obstructive pulmonary disease, and hepatitis B.

The clinical diagnosis and classification criteria were made according to the Novel Coronavirus Pneumonia Diagnosis and Treatment Plan (6 ${ }^{\text {th }}$ Edition) released by the National Health Commission of the People's Republic of China. The mild cases showed mild clinical symptoms, but absence of an obvious infection in imaging. The common cases were diagnosed from fever, cough, and local imaging. The severe cases were those, who met one of the following conditions: (1) respiratory distress, with respiratory rate of $\geqq 30$ times/min; (2) hypoxemia, with oxygen saturation $\leqslant 93 \%$ on finger pulse oximeter taken at rest; and (3) arterial partial pressure of oxygen $\left(\mathrm{PaO}_{2}\right) /$ fraction of inspired oxygen $\left(\mathrm{FiO}_{2}\right) \leqslant 300 \mathrm{~mm}$ $\mathrm{Hg}(1 \mathrm{~mm} \mathrm{Hg}=0.133 \mathrm{kPa})$. The critically ill cases met one of the following conditions: (1) respiratory failure, requiring mechanical ventilation; (2) shock; (3) combined failure of other organs [8,9]. In the present study, the mild and common cases were grouped as mild, and the severe and critically ill cases were grouped as severe.

\subsection{Imaging and lung function test}

The CT scans were conducted with a Siemens SOMATOM Force 64-slice spiral CT, and the spiral scans were conducted from the apex to the base of the lung, when the patient was asked to hold breath after maximum inspiration. The tube voltage was $120 \mathrm{kV}$, the tube current was $200 \mathrm{~mA}$, the slice thickness was $5 \mathrm{~mm}$, and the pitch was of 3.0. The image window width was $1000 \sim 1500 \mathrm{HU}$, and the window level was $-500 \sim-800 \mathrm{HU}$. Referring to the international scoring methods for idiopathic pulmonary fibrosis (IPF) lesions, the residual ground-glass opacification and interstitial shadows in the lungs (including interlobular septal thickening, subpleural curvilinear shadow, and irregular cords) were scored. Each residual lesion of lung lobe was scored from $0 \sim 5$ points (Table 1), and the total score for each medical sign was $0 \sim 25$ points.

The Minato AS-507 spirometer (Japan) was used to measure the following indicators for 192 recovered patients: the percentage of forced expiratory volume in one second to the predicted volume (FEV1.0\%), 
Table 1

System for scoring the groundglass opacity, interstitial opacity on thin-section CT scans

\begin{tabular}{cl}
\hline Score & \multicolumn{1}{c}{ Definition } \\
\hline 0 & None \\
1 & $<5 \%$ of the lobe \\
2 & $5-25 \%$ of the lobe \\
3 & $26-49 \%$ of the lobe \\
4 & $50-75 \%$ of the lobe \\
5 & $>75 \%$ of the lobe \\
\hline
\end{tabular}

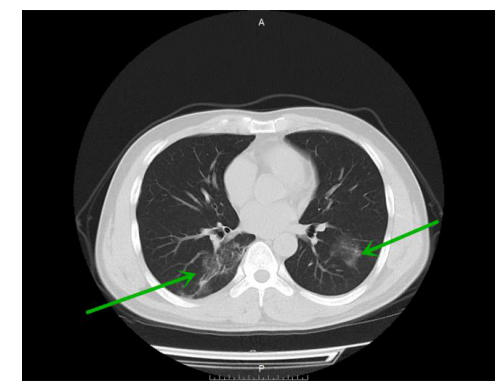

Fig. 1. Bilateral ground glass opacities with fuzzy edge in the lower lobes in a 35-year old woman (mild group).

the percentage of forced vital capacity to the predicted value (FVC\%), and the percentage of total lung capacity to the predicted value (TLC\%), and the percentage of single-breath diffusing capacity to the predicted value (DLCO $\%)$.

\subsection{Statistical analysis}

The symmetric distributed measurement data was represented by the mean \pm standard deviation (SD), while the skewed distributed measurement data was represented by the median. The comparison of the means between different groups was conducted by the Student's $t$-test or the Wilcoxon rank-sum test, and the comparison of the proportions between groups was conducted by the $\mathrm{X}^{2}$ test or Fisher's exact probability test. The logistic regression was used to analyze the risk factors of patients with severe illness and the ROC curve was made to analyze the correlation. The statistical software used was SPSS 22.0, and $P<0.05$ was considered statistically significantly different.

\section{Results}

\subsection{Primary symptoms}

There were 167 cases of fever with a temperature of $37.3-39^{\circ} \mathrm{C}(85.2 \%), 121$ cases of cough $(61.73 \%)$, 24 cases of phlegm (12.24\%), 59 cases of fatigue and muscle pain (30.1\%), 20 cases of anorexia (10.2\%), 15 cases of diarrhea (7.65\%), 9 cases of chest pain (4.59\%), and 5 cases of headache (2.55\%).

\subsection{General clinical data analysis}

There were 178 cases (90.81\%) with typical multiple patch shadows and ground-glass opacities in 


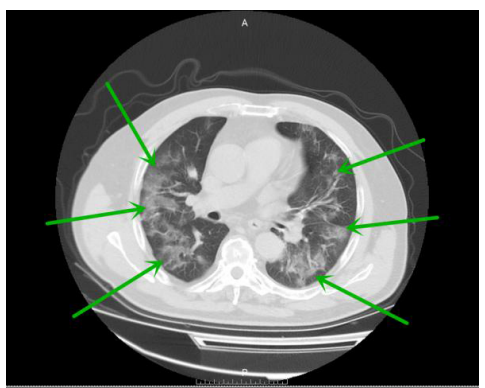

Fig. 2. Bilateral patchy infiltrates in the lower lung lobes in a 55-year old man (severe symptom group).
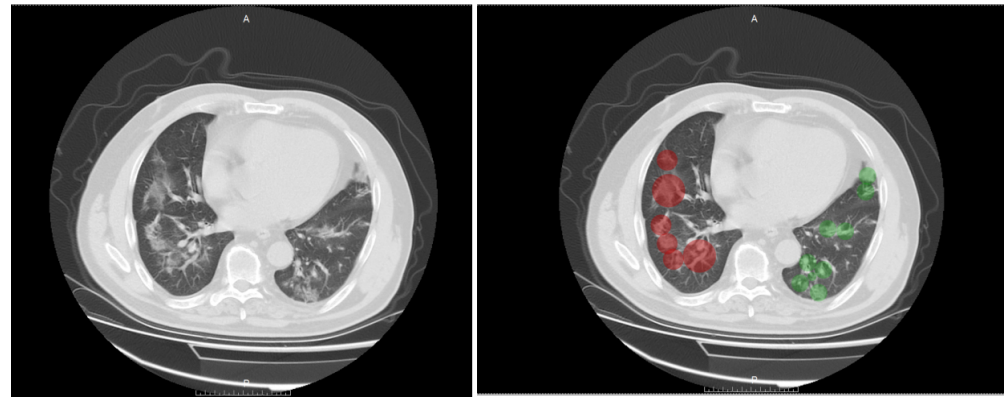

Fig. 3. CT visual score of severe COVID-19 pneumonia.

the first chest CT scan (Figs 1-3). The NCP laboratory test results were as follows: 30 cases were found to be with reduced white blood cells (WBC) (15.3\%), 110 cases with reduced lymphocytes (LY) $(55.6 \%), 80$ cases with reduced hemoglobin $(40.82 \%), 51$ cases with increased alanine aminotransferase (ALT) $(26.02 \%), 86$ cases with increased creatine kinase (CK) (43.88\%), 96 cases with increased lactate dehydrogenase (LDH) (48.98\%); 141 cases with increased c-reactive protein (CRP) (71.94\%), 122 cases with increased erythrocyte sedimentation rate (ESR) (62.24\%), and 59 cases with increased procalcitonin (PCT) $(30.1 \%)$.

\subsection{Clinical data analysis between the severe symptom group and the mild group}

There were 44 cases of severe illness and 152 cases of mild illness. There were significant differences in factors like age, gender, combined underlying disease, features of chest CT, WBC, LY, ESR, CRP, albumin, ALT, CK, LDH, and D-dimer (D-D) between these two groups $(P<0.05)$. On the other hand, there were no significant differences in epidemiological history, symptoms, platelet count, total bilirubin, aspartate aminotransferase (AST), creatinine, PCT, prothrombin time (PT), and electrolytes between these two groups $(P>0.05)$ (Table 2). All patients in the mild group recovered, while 4 patients in the severe group died of multiple organ failure, with a mortality rate of $2.04 \%$. After $10 \sim 63$ days of treatment, 192 patients recovered and were discharged, and further they were followed up for 30-128 days. The primary symptoms shown during the follow-up examinations included 22 cases of chest tightness, 15 cases of shortness of breath, 13 cases of fatigue, and 6 cases of joint pain. The CT re-examination results revealed 13 cases of fibrosis, 12 cases of interlobular septal thickening, and 3 cases of bronchiectasis.

\subsection{Analysis of risk factors for severe illness}

The univariate analysis indicated that age, gender, BMI, combined underlying diseases, chest CT 
Table 2

Clinical data analysis between the severe symptom group and the mild group

\begin{tabular}{|c|c|c|c|c|c|}
\hline General information & All cases $(n=196)$ & Severe group $(n=44)$ & Mild group $(n=152)$ & $t / X^{2}$ & $P$ value \\
\hline $\operatorname{Age}^{*}$ & $51.7 \pm 12.98$ & $60.18 \pm 10.13$ & $49.24 \pm 12.70$ & $t=10.938$ & $<0.001$ \\
\hline Gender* & & & & 5.76 & 0.0164 \\
\hline Male & 107 & 31 & 76 & & \\
\hline Female & 89 & 13 & 76 & & \\
\hline $\mathrm{BMI}^{*}$ & $22.23 \pm 2.19$ & $23.34 \pm 2.78$ & $21.92 \pm 1.89$ & 3.185 & 0.002 \\
\hline First nucleic acid test* & & & & 12.81 & $<0.001$ \\
\hline Positive & 127 & 34 & 71 & & \\
\hline Negative & 69 & 10 & 81 & & \\
\hline Epidemiology & & & & 0.0085 & 0.926 \\
\hline Yes & 117 & 26 & 91 & & \\
\hline No & 79 & 18 & 61 & & \\
\hline Smoking history & & & & 2.83 & 0.092 \\
\hline Yes & 29 & 10 & 19 & & \\
\hline No & 167 & 34 & 133 & & \\
\hline Underlying disease* & & & & 9.916 & 0.00164 \\
\hline Yes & 80 & 27 & 53 & & \\
\hline No & 116 & 17 & 99 & & \\
\hline Clinical symptoms & & & & & \\
\hline Fever & & & & 0.0605 & 0.80569 \\
\hline Yes & 167 & 38 & 129 & & \\
\hline No & & 6 & 23 & & \\
\hline Cough & & & & 0.485 & 0.317 \\
\hline Yes & 121 & 29 & 92 & & \\
\hline No & 75 & 15 & 60 & & \\
\hline Fatigue and myalgia & & & & 0.0794 & 0.778 \\
\hline Yes & 59 & 14 & 45 & & \\
\hline No & 137 & 30 & 107 & & \\
\hline Diarrhea & & & & 0.0073 & 0.931 \\
\hline Yes & 15 & 3 & 12 & & \\
\hline No & 137 & 41 & 140 & & \\
\hline Anorexia & & & & 0.0832 & 0.773 \\
\hline Yes & 20 & 5 & 15 & & \\
\hline No & 176 & 39 & 137 & & \\
\hline Chest CT findings* & & & & 4.41 & 0.035 \\
\hline No & 18 & 0 & 18 & & \\
\hline Yes & 178 & 44 & 135 & & \\
\hline Laboratory examination & & & & & \\
\hline $\mathrm{WBC}^{*}$ & $6.45 \pm 3.07$ & $7.85 \pm 3.4$ & $6.04 \pm 2.85$ & 3.212 & 0.002 \\
\hline $\mathrm{LC}^{*}$ & $0.72 \pm 0.29$ & $0.49 \pm 0.31$ & $0.78=0.25$ & -6.526 & $<0.001$ \\
\hline HB & $125.27 \pm 10.486$ & $123.89 \pm 8.224$ & $125.66 \pm 11.047$ & -0.99 & 0.323 \\
\hline PLT & $169.56 \pm 43.382$ & $177.7 \pm 45.58$ & $167.71 \pm 42.588$ & 1.418 & 0.158 \\
\hline ALB $^{*}$ & $37.76 \pm 6.25$ & $35.56 \pm 4.47$ & $38.39 \pm 6.55$ & -3.297 & 0.001 \\
\hline TB & $8.85 \pm 3.43$ & $9.43 \pm 2.8$ & $8.68 \pm 3.59$ & 1.271 & 0.205 \\
\hline ALT $^{*}$ & $30.28 \pm 14.99$ & $39.09 \pm 22.93$ & $27.74 \pm 10.55$ & 3.187 & 0.03 \\
\hline AST & $43.28 \pm 12.09$ & $33.03 \pm 25.13$ & $41.04 \pm 9.45$ & 6.133 & 0.65 \\
\hline $\mathrm{CK}^{*}$ & $299.49 \pm 137.32$ & $344.18 \pm 182.89$ & $297.16 \pm 114.25$ & 2.072 & 0.04 \\
\hline $\mathrm{LDH}^{*}$ & $288.71 \pm 71.75$ & $314.75 \pm 85.28$ & $281.17 \pm 65.74$ & 2.412 & 0.019 \\
\hline $\mathrm{CRP}^{*}$ & $20.54 \pm 14.76$ & $38.01 \pm 18.72$ & $15.48 \pm 8.16$ & 11.563 & $<0.001$ \\
\hline Creatinine & $70.01 \pm 16.87$ & $72.18 \pm 13.23$ & $69.38 \pm 17.77$ & 0.97 & 0.33 \\
\hline $\mathrm{K}$ & $3.93 \pm 0.44$ & $3.88 \pm 4.29$ & $3.95 \pm 4.39$ & -0.931 & 0.353 \\
\hline NA & $139.38 \pm 6.13$ & $138.06 \pm 7.29$ & $139 . \overline{77} \pm 5.72$ & -1.64 & 0.103 \\
\hline CL & $105.19 \pm 4.19$ & $104.83 \pm 3.36$ & $105.30 \pm 4.4$ & -0.646 & 0.519 \\
\hline $\mathrm{HCO} 3$ & $24.39 \pm 3.0$ & $24.769 \pm 3.21$ & $24.27 \pm 3.03$ & 0.937 & 0.35 \\
\hline $\mathrm{ESR}^{*}$ & $30.03 \pm 13.7$ & $35.45 \pm 19.38$ & $28.45 \pm 11.26$ & 2.287 & 0.026 \\
\hline PT & $10.38 \pm 1.15$ & $10.56 \pm 1.48$ & $10.33 \pm 1.03$ & 1.184 & 0.238 \\
\hline APTT & $30.82 \pm 5.33$ & $31.73 \pm 5.79$ & $30.56 \pm 5.18$ & 1.275 & 0.204 \\
\hline PCT & $0.78 \pm 0.83$ & $0.074 \pm 0.59$ & $0.079 \pm 0.89$ & -0.295 & 0.768 \\
\hline D-D* & $0.62 \pm 0.603$ & $1.03 \pm 0.87$ & $0.50 \pm 0.43$ & 5.445 & $<0.001$ \\
\hline
\end{tabular}

Note: * comparison between the two groups, $P<0.05$. 
Table 3

Analysis of risk factors in the severe symptom group

\begin{tabular}{|c|c|c|c|c|}
\hline \multirow[t]{2}{*}{ Variable quantity } & \multicolumn{2}{|c|}{ Single-factor regression analysis } & \multicolumn{2}{|c|}{ Multi-factor regression analysis } \\
\hline & $(95 \% \mathrm{CI})$ & $P$ value & $(95 \% \mathrm{CI})$ & $P$ value \\
\hline Age & $0.924(0.893-0.955)$ & $<0.001^{\#}$ & $0.961(0.778-0.952)$ & $0.004^{*}$ \\
\hline Gender & $0.419(0.204-0.863)$ & $0.018^{\#}$ & $3.549(0.453-27.804)$ & 0.228 \\
\hline BMI & $0.748(0.639-0.875)$ & $<0.001^{\#}$ & $0.624(0.409-0.950)$ & $0.028^{*}$ \\
\hline First nucleic acid test & $0.464(0.213-1.008)$ & 0.052 & & \\
\hline Epidemiology & $1.033(0.522-2.045)$ & 0.926 & & \\
\hline Smoking history & $0.486(0.207-1.140)$ & 0.097 & & \\
\hline Underlying disease & $0.337(0.169-0.674)$ & $0.002^{\#}$ & $4.172(1.271-13.691)$ & $0.019^{*}$ \\
\hline Clinical symptoms & & & & \\
\hline Fever & $0.886(0.336-2.333)$ & 0.806 & & \\
\hline Cough & $0.793(0.939-1.602)$ & 0.518 & & \\
\hline Fatigue and myalgia & $0.901(0.437-1.858)$ & 0.778 & & \\
\hline Diarrhea & $1.171(0.315-4.351)$ & 0.813 & & \\
\hline Anorexia & $0.854(0.292-2.497)$ & 0.773 & & \\
\hline Hospital stay & $1.193(0.944-1.212)$ & $0.011^{\#}$ & $0.893(0.902-1.302)$ & $0.003^{*}$ \\
\hline Chest CT findings & $2.170(2.831-3.452)$ & $<0.001^{\#}$ & $1.005(0.673-1.143)$ & $0.002^{*}$ \\
\hline Laboratory examination & & & & \\
\hline WBC & $0.722(0.64-0.816)$ & $0.001^{\#}$ & $1.25(0.883-1.770)$ & 0.208 \\
\hline $\mathrm{LC}$ & $5.739(43.26-260.18)$ & $<0.001^{\#}$ & $8.901(2.352-33.693)$ & $0.002^{*}$ \\
\hline HB & $1.018(0.983-1.053)$ & 0.32 & & \\
\hline PLT & $0.995(0.987-1.002)$ & 0.159 & & \\
\hline ALB & $1.091(1.021-1.166)$ & $0.01^{\#}$ & $1.017(0.871-1.1188)$ & 0.828 \\
\hline TB & $1.223(0.928-1.033)$ & 0.189 & & \\
\hline ALT & $0.953(0.931-0.976)$ & $0.003^{\#}$ & $0.984(0.925-1.047)$ & 0.606 \\
\hline AST & $0.986(0.959-1.013)$ & 0.314 & & \\
\hline CK & $0.997(0.994-1.000)$ & $0.029^{\#}$ & $0.996(0.987-1.004)$ & 0.298 \\
\hline LDH & $0.993(0.988-0.998)$ & $0.007^{\#}$ & $1.007(0.901-1.018)$ & $0.026^{*}$ \\
\hline CRP & $0.201(0.111-0.362)$ & 0.001 & $0.866(0.790-0.948)$ & $0.002^{*}$ \\
\hline Creatinine & $0.955(0.782-1.166)$ & 0.651 & & \\
\hline $\mathrm{K}$ & $1.441(0.663-3.11)$ & 0.352 & & \\
\hline NA & $1.043(0.991-1.098)$ & 0.108 & & \\
\hline CL & $1.026(0.950-1.107)$ & 0.519 & & \\
\hline $\mathrm{HCO} 3$ & $0.949(0.849-1.059)$ & 0.349 & & \\
\hline ESR & $0.967(0.945-0.990)$ & 0.004 & $0.974(0.919-1.032)$ & 0.370 \\
\hline PT & $0.847(0.642-1.117)$ & 0.24 & & \\
\hline APTT & $0.960(0.901-1.023)$ & 0.204 & & \\
\hline PCT & $1.953(0.023-166.598)$ & 0.768 & & \\
\hline D-D & $0.979(0.959-0.999)$ & 0.039 & $0.536(0.138-2.081)$ & 0.368 \\
\hline CT visual score & $0.997(0.994-1.000)$ & 0.029 & $0.996(0.987-1.004)$ & $0.298^{*}$ \\
\hline TGGO & $0.993(0.988-0.998)$ & 0.007 & $1.007(0.901-1.018)$ & $0.265^{*}$ \\
\hline IOF & $1.043(0.991-1.098)$ & 0.108 & & \\
\hline
\end{tabular}

Note: ${ }^{*} P<0.05$.

features, CT visual score, WBC, absolute lymphocyte count (ALC), albumin, ALT, CK, LDH, CRP, ESR and D-D were the risk factors of severe illness. The multivariate logistic regression analysis revealed that age, underlying disease, length of hospital stay, BMI, LDH, chest CT visual score, ALC, and CRP were risk factors for severe illness (Table 3). The ROC curve demonstrated that the chest CT visual score was the most valuable indicator for predicting severe illness. With a CT visual score of $<8.97$ as the cut-off, the severe illness prediction had a sensitivity of $93.22 \%$ and a specificity of $71.53 \%$, and it was negatively correlated with prognostic DLCO $\%$ with a coefficient of 0.702 , followed by IOF (Table 4 and Fig. 4). 
Table 4

Results of ROC curve of clinical imaging parameters in the severe symptom group

\begin{tabular}{lcccc}
\hline \multicolumn{1}{c}{ Indexes } & Area under curve & Critical value & Sensitivity (\%) & Specificity (\%) \\
\hline CT visual score & 0.912 & 8.97 & 93.22 & 71.53 \\
TGGO & 0.892 & 3.591 & 86.90 & 53.10 \\
Chest CT findings & 0.772 & 2.155 & 85.10 & 35.60 \\
CRP & 0.622 & 3.625 & 78.10 & 38.10 \\
LC & 0.552 & 1.445 & 50.60 & 71.20 \\
Underlying disease & 0.432 & 0.745 & 53.80 & 64.50 \\
\hline
\end{tabular}

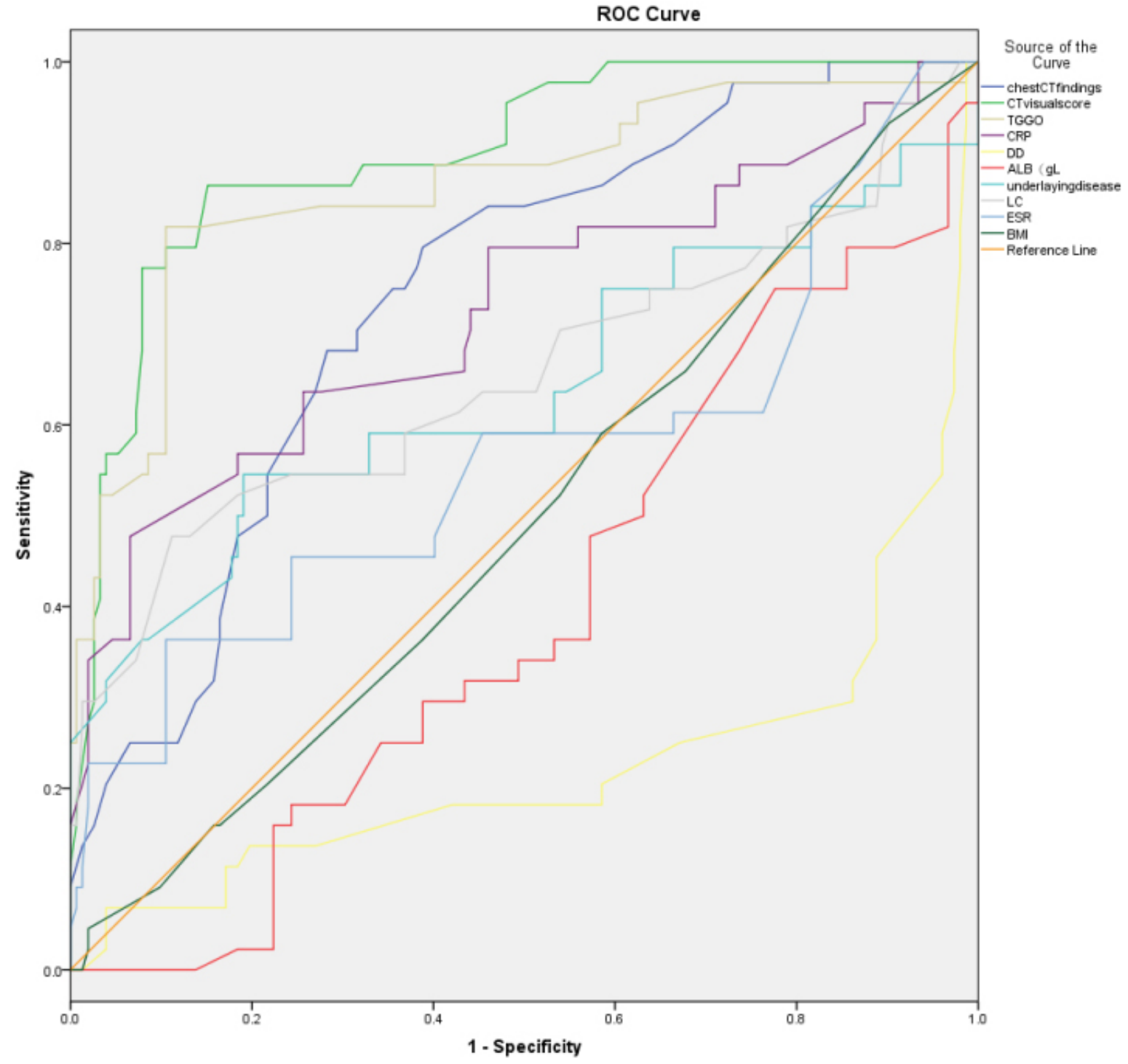

Fig. 4. Results of the ROC curve of the risk factors of the Severe SARS-CoV-2 Pneumonia; The results of this study also show that "CT visual score" is the largest area under the ROC curve of the prognosis of pneumonia, and its diagnostic value of predicting EA fetus is the highest. The sensitivity was $93.22 \%$ and the specificity was $71.53 \%$. 
Table 5

Correlation between the CT visual score and $\mathrm{FVC} \%$, TLC\%, DLCO \% (correlation coefficent: r)

\begin{tabular}{llll}
\hline & FVC\% & TLC\% & DLCO $\%$ \\
\hline TGGO & -0.227 & -0.157 & -0.383 \\
IOF & -0.502 & -0.458 & $-0.697 \#$ \\
CT visual score & -0.513 & -0.494 & $-0.702 \#$ \\
\hline
\end{tabular}

\subsection{Follow-up of recovered patients}

We followed up 192 recovered patients for $1 \sim 4$ months. It was found that the degree of pulmonary fibrosis in NCP patients directly affected the lung function indicators. Furthermore, the CT visual score was negatively correlated with FEV1.0\%, FVC\%, TLC\%, and DLCO\% (Table 5).

\section{Discussion}

SARS-CoV-2 is a novel coronavirus of the $\beta$ genus, shaped round or elliptic, with a spike-like structure wrapping around the virus capsid and showing a crown-like appearance under an electron microscope. The homology between SARS-CoV-2 and the bat SARS-like coronavirus (BatCoVRaTG13) is as high as $96.3 \%$, and bats may be its potential natural host $[10,11]$. The primary symptoms of NCP are fever, dry cough and fatigue, and $25.2 \%$ of patients may have difficulty breathing. The severe case may progress to acute respiratory distress syndrome, septic shock, and multiple organ failure. It has been reported that the SARS-Cov-2 has a strong affinity for human respiratory receptors, implying a potential threat to global public health [12-14].

Among the 196 cases of NCP in this study, there were 107 males and 89 females (1.2:1). The ratio of male to female, BMI, and age of the severe group were greater than those of the mild group (31 vs. 13, $23.34 \pm 2.78$ vs. $21.92 \pm 1.89$, and $60.18 \pm 10.13$ vs. $49.24 \pm 12.70$, respectively), probably related to the weakened immunity of elderly obese patients $[15,16]$. In addition, 117 cases of NCP had a history of contact with confirmed patients, and 80 cases had underlying diseases. While, only 137 cases were first-round laboratory tested positive, indicating a low sensitivity of single round nucleic acid test for the NCP diagnosis, probably due to low viral load, non-standard sampling methods, or poor quality of reagents. Therefore, multiple nucleic acid screening tests or supplementary examination with serum antibodies are needed for the effective control of the disease transmission $[17,18]$. We also found that the first-round nucleic acid test positive rate of the severe group was much higher than that of the mild group (77.27\% vs. $46.71 \%)$, confirming the necessity of screening of the severe illness, prevention, and special care of the patients with first-round positive nucleic acid results. The primary symptoms of NCP are fever and dry cough. The fever ratio in NCP patients in this study was $85.2 \%$, mostly wave fever $(46.2 \%)$ and intermittent fever $(25.1 \%$ ), with only a few high fever cases (13.9\%), suggesting needs for increasing screening for patients with wave fever and intermittent fever. There were $14.8 \%$ of NCP patients with no fever, higher than the previously reported SARS and MERS infections. Hence, asymptomatic infections cannot be ignored to avoid missed diagnosis $[19,20]$. There were 94 cases of NCP with symptoms such as fatigue, muscle pain, and anorexia, probably due to the lower respiratory tract being the target of SARS-Cov-2, and special attention should be paid to such cases.

The CT results of 178 cases $(90.82 \%)$ of NCP were positive, therefore, the chest CT was highly sensitive to SARS-Cov-2 pneumonia [21,22]. In addition, we newly introduced a CT visual scoring mechanism to evaluate the progress of disease and obtain good results. At the same time, multivariate 
analysis confirmed that $\mathrm{CT}$ score was an important parameter related to the prognosis of NCP. The CT signs of NCP are ground-glass opacifications and interstitial shadows (including interlobular septal thickening, subpleural curvilinear shadow, and irregular cords). Nevertheless, the lung fiber cord shadows, interlobular septal thickening, subpleural curvilinear shadow generally do not appear alone, but on the top of ground-glass opacifications. The ground-glass opacifications in the mild group were gradually absorbed and became lighter, especially for those with lesions involving less than two lung lobes, lung segments and low-density lesions. While a small portion of the ground-glass consolidations, cord shadows and subpleural curvilinear shadows could be absorbed too in the severe group. However, it was difficult for the dense cord shadows to be absorbed and showing no changes in the lesions even at the 3-month follow-up. The results were good to quantify the difference between the two groups with CT visual score, with significantly bigger scores in the severe group than in the mild group (8.53 \pm 2.36 vs. $5.11 \pm 1.96)$. In the past, it was difficult to achieve a standard using the CT signs based on human subjective opinions, making it difficult to tell the differences between severe and mild cases as well as the prognosis [23-25]. CT visual scores perfectly solved this dilemma.

According to reports, lung parenchyma, irregular interface signs, and traction bronchiectasis are the signs of fibrosis, which are related to the patient's age and gender, i.e. the elderly male patients are more likely to have persistent fibrotic lesions in the lungs [26-28]. In the present study, it was found that the ground-glass shadows and reticular shadows were common in survivors with severe NCP. The peak value of LDH indicated the degree of tissue damage. The high peak values of LDH were also seen in the patients with higher visual scores of residual lung lesions. The more residual shadows in the lungs, the bigger declines in TLC\% and DLCO\% in the lung function follow-up examinations. The residual lung lesions were negatively correlated with DLCO\%. The CT visual score strikingly quantified the relationship between these two, and the image performance and the recovery of lung function were closely related to the clinical manifestations [29-31].

\section{Conclusions}

In the follow-up after NCP recovery, it was shown that the lung CT features were closely related to its clinical manifestations, laboratory tests and lung function. There was a gradual improvement in the residual lung lesions and lung function, but the absorption of the interstitial shadows was slow. CT is able to demonstrate even the minute changes in the lungs, thus is an ideal examination method for monitoring residual lesions in lungs. Pulmonary function test is of great significance in evaluating the prognosis and quality of life of patients. Nevertheless, a longer follow-up is expected to check the recovery of residual lesions in lungs, especially the interstitial shadows in the NCP survivors, and the imaging performance of NCP needs to be further explored and studied.

\section{Acknowledgments}

This work was supported by grants from Hubei Province Health and Family Planning Scientific Research Project (No. WJ2019F096 and No. WJ2019F092), the Scientific Research Project of Hubei University of Medicine (Xiangyang No. 1 People's Hospital): Application of Comparative Imaging Combined with Problem-based Learning (PBL) in Medical Imaging Theory Teaching (Zhibing He); the Study on the Diagnostic Value of Digital Mammography and DCE-MRI Combined with Serum-related Tumor Marker Detection in Clinical Palpation-negative Breast Cancer (Zhibing He); and Comparison of the Composition of Intestinal Microbiota between Patients with Colon Adenoma and Colon Cancer by High-throughput Sequencing (Yingjian Ye). 


\section{Conflict of interest}

The authors declare no conflict of interest.

\section{References}

[1] Van Caeseele P, Bailey D, Forgie SE, Dingle TC, Krajden M. SARS-CoV-2 (COVID-19) serology: implications for clinical practice, laboratory medicine and public health [published online ahead of print, 2020 Aug 3]. CMAJ. 2020; cmaj.201588. doi: 10.1503/cmaj.201588.

[2] Tan CS, Yeoh SF, Long CM. COVID-19: critical role of angiotensin 1-7 in ACE2 modulation. Ann Acad Med Singapore. 2020 Jun; 49(6): 398-400. PMID: 32712639.

[3] Lu R, Zhao X, Li J, et al. Genomic characterisation and epidemiology of 2019 novel coronavirus: implications for virus origins and receptor binding. Lancet. 2020; 395(10224): 565-574. doi: 10.1016/S0140-6736(20)30251-8.

[4] World Health Organization. WHO Coronavirus Disease (COVID-19) Dashboard (https://www.who.int. opens in new tab).

[5] World Health Organization. Coronavirus disease (COVID-19) outbreak (https://www.who.int. opens in new tab).

[6] Ai T, Yang Z, Hou H, Zhan C, Chen C, Lv W, et al. Correlation of chest CT and RT-PCR testing for coronavirus disease 2019 (COVID-19) in China: a report of 1014 cases. Radiology. 2020 Aug; 296(2): E32-E40. doi: 10.1148/ radiol.2020200642. Epub 2020 Feb 26. PMID: 32101510; PMCID: PMC7233399.

[7] Antonio GE, Wong KT, Hui DS, et al. Thin-section CT in patients with severe acute respiratory syndrome following hospital discharge: preliminary experience. Radiology. 2003; 228(3): 810-815. doi: 10.1148/radiol.2283030726.

[8] General Office of the National Health and Health Commission. Notice on Issuing a New Coronavirus Pneumonia Diagnosis and Treatment Plan (Trial Implementation of Revised Fifth Edition). National Health Office Medical Letter [2020] No. 117. 2020. http://www.nhc.gov.cn/yzygj/s7653p/202002/3b09b894ac9b4204a79db5b8912d4440.shtml.

[9] Goh KJ, Choong MC, Cheong EH, Kalimuddin S, Duu Wen S, Phua GC, et al. Rapid progression to acute respiratory distress syndrome: review of current understanding of critical illness from COVID-19 infection. Ann Acad Med Singapore. 2020 Mar 16; 49(3): 108-118. PMID: 32200400.

[10] Abu-Raya B, Gantt S, Sadarangani M. Challenges in evaluating SARS-CoV-2 vaccines during the COVID-19 pandemic [published online ahead of print, 2020 Jul 9]. CMAJ. 2020; cmaj.201237. doi: 10.1503/cmaj.201237.

[11] Xu Z, Shi L, Wang Y, Zhang J, Huang L, Zhang C, et al. Pathological findings of COVID-19 associated with acute respiratory distress syndrome. Lancet Respir Med. 2020 Apr; 8(4): 420-422. doi: 10.1016/S2213-2600(20)30076-X. Epub 2020 Feb 18. Erratum in: Lancet Respir Med. 2020 Feb 25; PMID: 32085846; PMCID: PMC7164771.

[12] Ye Z, Rochwerg B, Wang Y, et al. Treatment of patients with nonsevere and severe coronavirus disease 2019: an evidence-based guideline. CMAJ. 2020; 192(20): E536-E545. doi: 10.1503/cmaj.200648.

[13] Frost DW, Shah R, Melvin L, et al. Principles for clinical care of patients with COVID-19 on medical units. CMAJ. 2020; 192(26): E720-E726. doi: 10.1503/cmaj.200855.

[14] Parhar KKS, Lequier L, Blackwood J, Zuege DJ, Singh G. Optimizing provision of extracorporeal life support during the COVID-19 pandemic: practical considerations for Canadian jurisdictions. CMAJ. 2020; 192(14): E372-E374. doi: 10.1503/cmaj.200448.

[15] Cheng MP, Lee TC, Tan DHS, Murthy S. Generating randomized trial evidence to optimize treatment in the COVID-19 pandemic. CMAJ. 2020; 192(15): E405-E407. doi: 10.1503/cmaj.200438.

[16] Bhandari S, Sharma S, Bhargava A, Keswani P, Sharma R, Shekhawat A. Inflammatory markers in COVID-19. Ann Acad Med Singapore. 2020 Jun; 49(6): 393-397. PMID: 32712638.

[17] Kumar DS, O'Neill SB, Johnston JC, Grant JM, Sweet DD. SARS-CoV-2 infection in a 76-year-old man with initially negative nasopharyngeal swabs. CMAJ. 2020; 192(20): E546-E549. doi: 10.1503/cmaj.200641.

[18] An P, Ye Y, Chen M, Chen Y, Fan W, Wang Y. Management strategy of novel coronavirus (COVID-19) pneumonia in the radiology department: a Chinese experience. Diagn Interv Radiol. 2020 May; 26(3): 200-203. doi: 10.5152/dir.2020.20167. PMID: 32209526; PMCID: PMC7239366.

[19] Nicholls JM, Poon LL, Lee KC, Ng WF, Lai ST, Leung CY, et al. Lung pathology of fatal severe acute respiratory syndrome. Lancet. 2003 May 24; 361(9371): 1773-8. doi: 10.1016/s0140-6736(03)13413-7. PMID: 12781536; PMCID: PMC7112492.

[20] Johkoh T, Müller NL, Taniguchi H, Kondoh Y, Akira M, Ichikado K, et al. Acute interstitial pneumonia: thin-section CT findings in 36 patients. Radiology. 1999 Jun; 211(3): 859-63. doi: 10.1148/radiology.211.3.r99jn04859. PMID: 10352616.

[21] An P, Wood BJ, Li W, Zhang M, Ye Y. Postpartum exacerbation of antenatal COVID-19 pneumonia in 3 women. CMAJ. 2020; 192(22): E603-E606. doi: 10.1503/cmaj.200553.

[22] An P, Liu B. Computed tomography manifestations of COVID-19 pneumonia. Br J Hosp Med (Lond). 2020; 81(4): 1-2. doi: 10.12968/hmed.2020.0104. 
[23] An P, Song P, Lian K, Wang Y. CT manifestations of novel coronavirus pneumonia: a case report. Balkan Med J. 2020; 37(3): 163-165. doi: 10.4274/balkanmedj.galenos.2020.2020.2.15.

[24] An P, Zhang M. Novel coronavirus SARS-CoV-2: familial spread resulting in COVID-19 pneumonia in a pediatric patient. Diagn Interv Radiol. 2020; 26(3): 262-263. doi: 10.5152/dir.2020.20157.

[25] An P, Song P, Wang Y, Liu B. Asymptomatic patients with novel coronavirus disease (COVID-19). Balkan Med J. 2020; 37(4): 229-230. doi: 10.4274/balkanmedj.galenos.2020.2020.4.20.

[26] Juurlink DN. Safety considerations with chloroquine, hydroxychloroquine and azithromycin in the management of SARS-CoV-2 infection. CMAJ. 2020; 192(17): E450-E453. doi: 10.1503/cmaj.200528.

[27] Rohailla S, Ahmed N, Gough K. SARS-CoV-2 infection associated with spontaneous pneumothorax. CMAJ. 2020; 192(19): E510. doi: 10.1503/cmaj.200609.

[28] Kirtsman M, Diambomba Y, Poutanen SM, et al. Probable congenital SARS-CoV-2 infection in a neonate born to a woman with active SARS-CoV-2 infection. CMAJ. 2020; 192(24): E647-E650. doi: 10.1503/cmaj.200821.

[29] Arya A, Buchman S, Gagnon B, Downar J. Pandemic palliative care: beyond ventilators and saving lives. CMAJ. 2020; 192(15): E400-E404. doi: 10.1503/cmaj.200465.

[30] Ng-Kamstra J, Stelfox HT, Fiest K, Conly J, Leigh JP. Perspectives on personal protective equipment in acute care facilities during the COVID-19 pandemic. CMAJ. 2020; 192(28): E805-E809. doi: 10.1503/cmaj.200575.

[31] Mahammedi A, Saba L, Vagal A, et al. Imaging in Neurological Disease of Hospitalized COVID-19 Patients: An Italian Multicenter Retrospective Observational Study [published online ahead of print, 2020 May 21]. Radiology. 2020; 201933. doi: 10.1148/radiol.2020201933. 